\title{
3-D transponder antennas for future SHF RFID applications
}

\author{
R. Zichner ${ }^{1}$ and R. R. Baumann ${ }^{1,2}$ \\ ${ }^{1}$ Fraunhofer Institute for Electronic Nano Systems ENAS, Department Printed Functionalities, Germany \\ ${ }^{2}$ Chemnitz University of Technology, Institute for Print and Media Technology, Germany
}

\begin{abstract}
The radio frequency identification (RFID) technology is omnipresent since a few years. Some of the most popular fields of application are the use for security tasks, for logistics and for the consumer segment. For example, chip card or key ring sized RFID transponders can allow wireless access to secured rooms. The number of applications for wireless data transmission for the identification and tracking of objects increases every year. There is a large development need for highly functional and inexpensive RFID transponders due to the ever-increasing demand on improved reliability, higher data rates and read and write ranges of the RFID systems. Therefore, research was performed on new 3D transponder antennas for the Super High Frequency Band around $5.8 \mathrm{GHz}$. Additionally, wave propagation effects and the influence of different dielectric environments were considered. Parallel to the design of the novel antenna structures, the printing process for inexpensive manufacturing was investigated. The gained results are the basis for prospective RFID applications.
\end{abstract}

\section{Introduction}

State of the art passive RFID transponder for long range application $(8 \mathrm{~m}-10 \mathrm{~m})$ operate in frequency ranges of $868 \mathrm{MHz}-928 \mathrm{MHz}$ and $2.45 \mathrm{GHz}$. Such transponders typically consist of a dipole antenna and a silicon RFID chip. In future communication technologies, these transponders are going to be disadvantaged due to the nondirectional typical dipole radiation characteristics (comp. Fig. 1):

- Lower maximum antenna gain $(2-4 \mathrm{dBi})$ because of nondirectional radiation

- High influence of dielectric environments (scattering, diffraction, reflection of electromagnetic radiation in dielectric medium) on transponder antenna characteristics

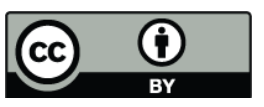

Correspondence to: R. Zichner (ralf.zichner@enas.fraunhofer.de)
- Limited ability to communicate in different defined directions

In order to increase the communication quality of passive RFID transponder systems so that they can be used in diverse applications like for example the Internet of Things (Bullinger and ten Hompel, 2007), it is necessary to design new transponder antenna structures. These structures need to have a directional radiation to do both minimizing the influence of surrounding dielectric materials and allowing more effective communication in defined directions. Furthermore, it is required to provide a broad frequency range for the transmission of huge amounts of data as well as to guarantee read and write ranges of more than $10 \mathrm{~m}$. Because of these requirements, transponder antennas in the frequency ranges of $5.725-5.875 \mathrm{GHz}$ (center frequency $5.8 \mathrm{GHz}$ ) are developed. Additionally, a very inexpensive, effective and flexible production process for producing transponder antenna is used to allow address numerous RFID applications.

\section{Basics}

Dipole antennas in their most elementary form have a linear symmetric structure. The electromagnetic fields which are generated by dipole antennas allow a Donat shaped radiation characteristic (comp. Fig. 1).

A standard dipole with half-wave length $(l=\lambda / 2)$ can be numerically divided in a variety of infinitesimal dipoles of a length $d z^{\prime}$ (comp. Fig. 2). For each of these infinitesimal dipoles, an electrical field distribution $d \boldsymbol{E}_{\theta}$ can be assigned (Balanis, 2005),

$d \boldsymbol{E}_{\theta} \cong j \eta \frac{k I_{\mathrm{e}} e^{-j k r}}{4 \pi r} \sin \theta e^{j k z^{\prime} \cos \theta} d z^{\prime}$

with

$I_{\mathrm{e}}\left(x^{\prime}=0, y^{\prime}=0, z^{\prime}\right)=\left\{\begin{array}{l}\hat{a}_{z} I_{0} \sin \left[k\left(\frac{l}{2}-z^{\prime}\right)\right], 0 \leq z^{\prime} \leq l / 2 \\ \hat{a}_{z} I_{0} \sin \left[k\left(\frac{l}{2}+z^{\prime}\right)\right],-l / 2 \leq z^{\prime} \leq 0\end{array}\right.$

and $k=2 \pi / \lambda$.

Published by Copernicus Publications on behalf of the URSI Landesausschuss in der Bundesrepublik Deutschland e.V. 


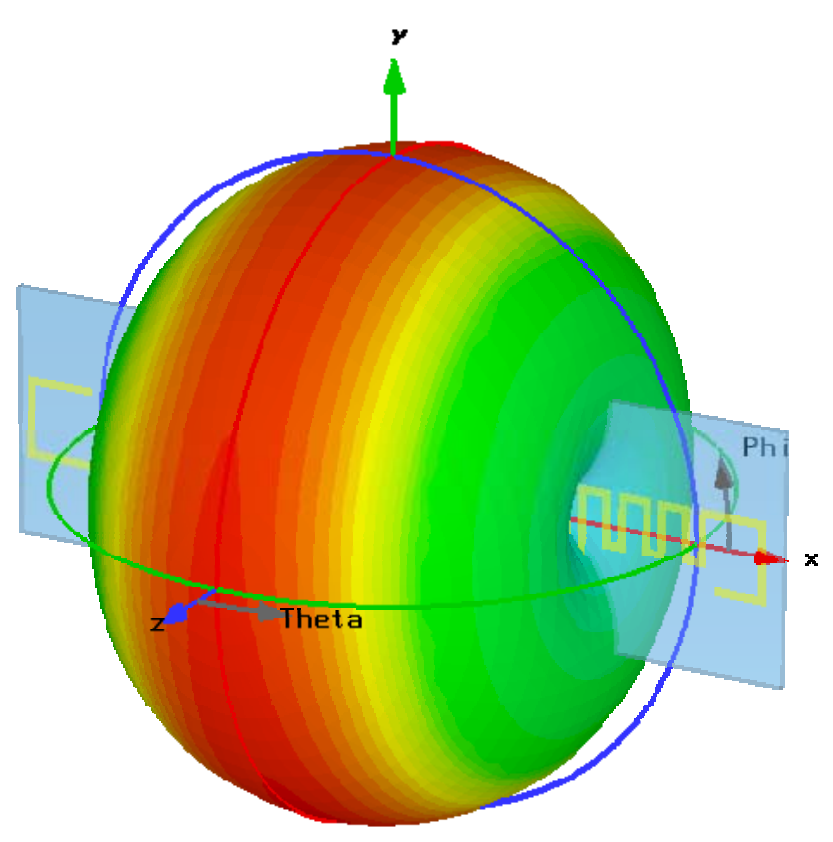

Fig. 1. Typical dipole radiation characteristic.

The sum of all dipoles results in the field distribution of the whole dipole.

$\boldsymbol{E}_{\theta}=\int_{-l / 2}^{+l / 2} d \boldsymbol{E}_{\theta}=j \eta \frac{k e^{-j k r}}{4 \pi r} \sin \theta\left[\int_{-l / 2}^{+l / 2} I_{e} e^{j k z^{\prime} \cos \theta} d z^{\prime}\right]$

This equation can be solved numerically:

$\boldsymbol{E}_{\theta} \cong j \eta \frac{I_{0} e^{-j k r}}{2 \pi r}\left[\frac{\cos \left(\frac{k l}{2} \cos \theta\right)-\cos \left(\frac{k l}{2}\right)}{\sin \theta}\right]$.

For a dipole with half-wave length $(l=\lambda / 2)$, the solution is as follows:

$$
\left|\boldsymbol{E}_{\theta}\right| \cong j \eta \frac{I_{0} e^{-j k r}}{2 \pi r}\left[\frac{\cos \left(\frac{\pi}{2} \cos \theta\right)}{\sin \theta}\right]
$$

Equation (5) shows the radiation characteristic of a standard half-wave length dipole. If, in contrast to this, a dipole structure is not designed symmetrically (comp. Fig. 3), significant deviations occur compared to standard dipoles. This topic is covered below.

\section{Results}

In order to implement the requirements for new transmission systems (transponder antennas) explained in the introduction, an adapted antenna structure was designed. This new antenna structure was dimensioned in its form and shape (comp. Fig. 3) with regard to a variety of parameters:

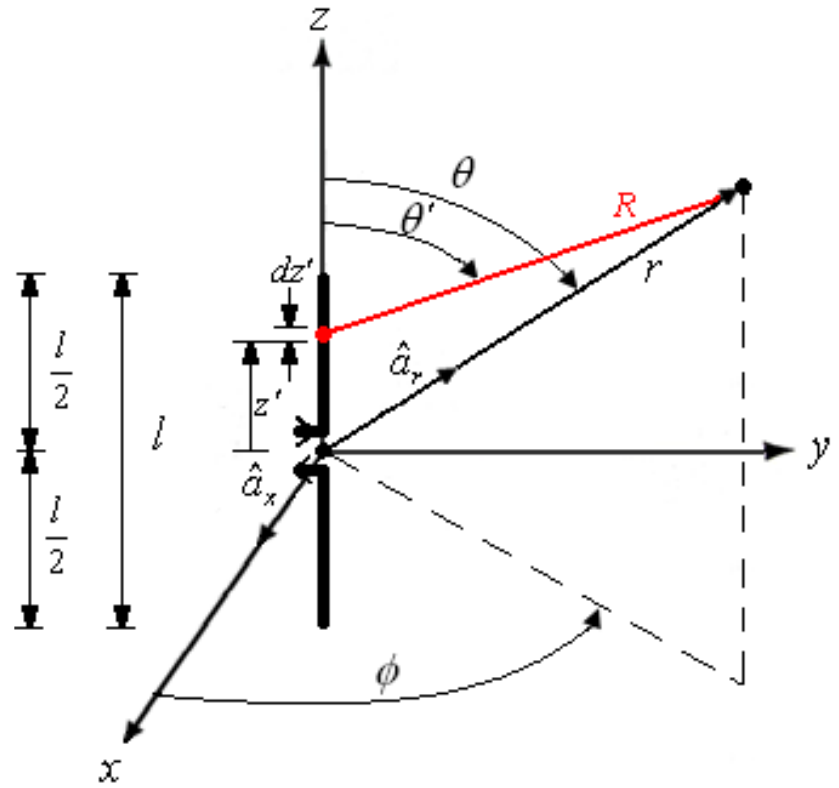

Fig. 2. Dipole and geometry.

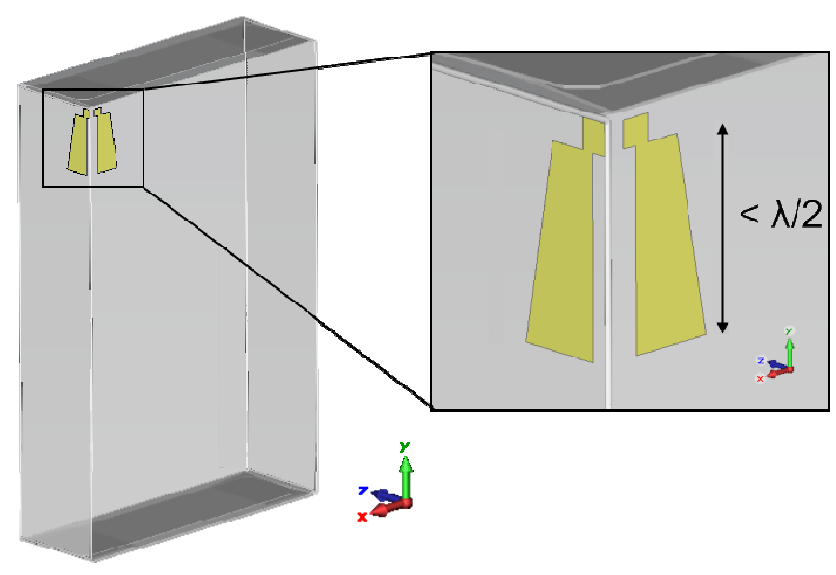

Fig. 3. Designed 3-D dipole-antenna structure.

The dipole arms shown in Fig. 3 are running parallel to each other and they are situated on levels shifted by $90^{\circ}$. Because of the parallel arrangement of the dipole arms, the antenna structure does not have a dipole typical radiation characteristic. The emerging radiation characteristic runs directional (comp. Fig. 4).

The directivity as well as the resonance frequency and the impedance adjustment are significantly influenced by the arrangement of the dipole arms which are shifted by $90^{\circ}(\alpha)$. The antenna structure was with regard to the $90^{\circ}$ arrangement of the dipole arms dimensioned and adapted. Changes of the three dimensional direction of the antenna arms (comp. Fig. 5) result in changes of the antenna characteristics.

From the scattering parameter (results of the simulation) shown in Fig. 6, it is obvious that with increasing as well as 

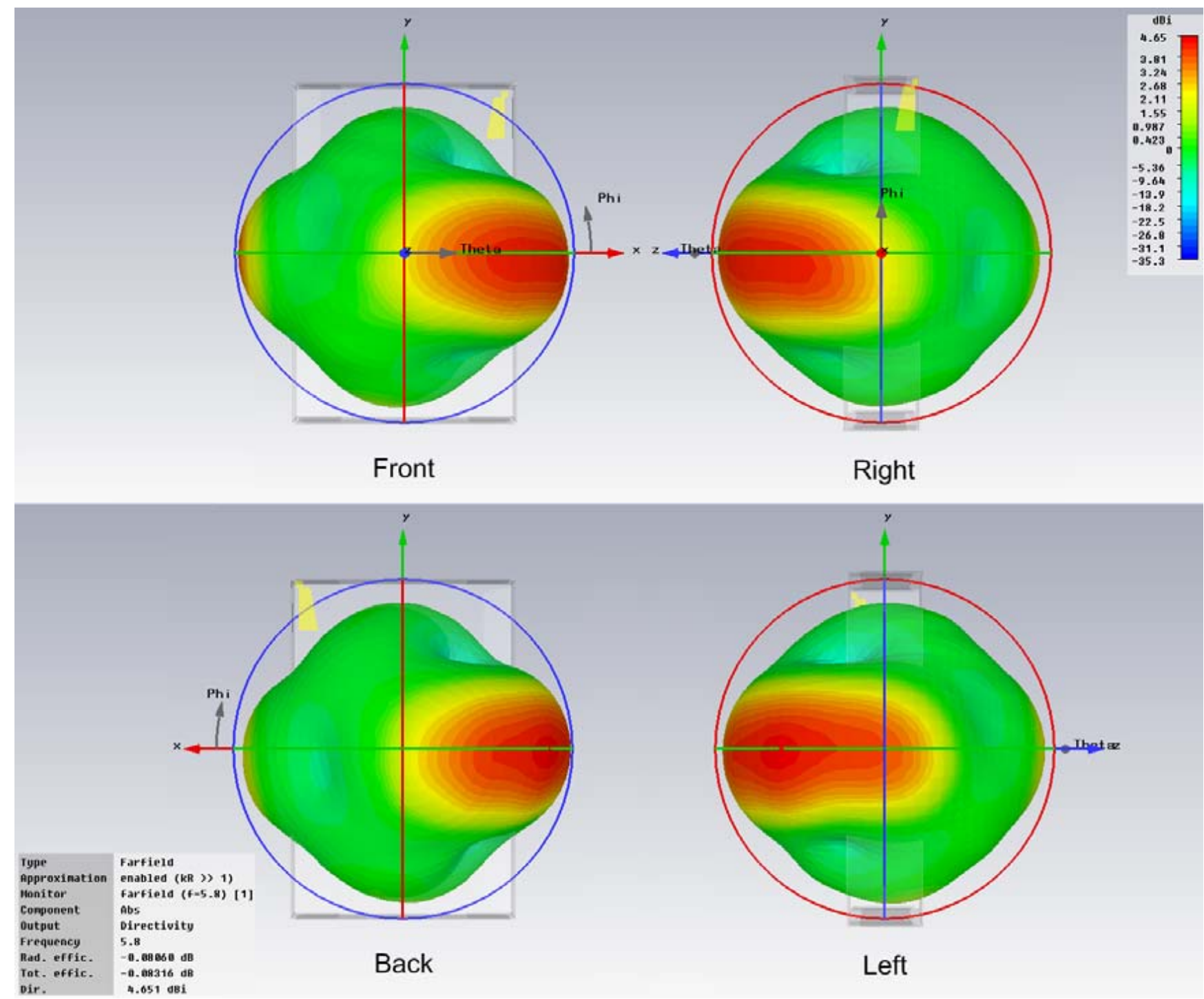

Fig. 4. 3-D directivity plot (CST microwave studio simulation).

diminishing angles $\alpha\left(40^{\circ}<90^{\circ}>180^{\circ}\right)$ of the dipole arms to one another, a clear shift of the resonance frequency and, at the same time, a decrease of the back scattering attenuation occurs. Despite that, the antenna structure in the angle range of $\alpha=80^{\circ}$ to $\alpha=100^{\circ}$ have the needed good antenna characteristics. But, as mentioned above, next to the scattering parameter, the three dimensional direction (angle $\alpha$ ) of the dipole arms as well as the directivity influence the characteristics.

The angle dependent variation of the directivity shown in Fig. 7 illustrates that with a decreasing angle $\alpha$, the directional radiation in main beam direction $\left(\mathrm{Phi}=0^{\circ}, \mathrm{Phi}=180^{\circ}\right)$ increases.

\subsection{Antenna manufacturing}

Today, planar standard RFID transponder antennas are still produced in a lithographic method. Because of the wetchemical etching process, this method is a comparatively time and cost intensive manufacturing process. Alternative manufacturing processes could be printing processes such as screen printing, inkjet or gravure printing. Those additive processes allow the application of structured conductive layer on different substrates (foil, paper, etc.). For the developed dipole antenna structure, screen printing (EKRA E2XL Screen Printer) was used. The antenna structure was printed on a planar paper substrate $(250 \mu \mathrm{m}$ thick, uncoated, permittivity $\varepsilon_{\mathrm{r}}=2.31$ ) with silver ink (SunChemical CRSN2442, sheet resistance: $0.01 \Omega / \mathrm{Sq}$ ). The used screen in combination with the silver ink allowed a printed silver layer thickness of 


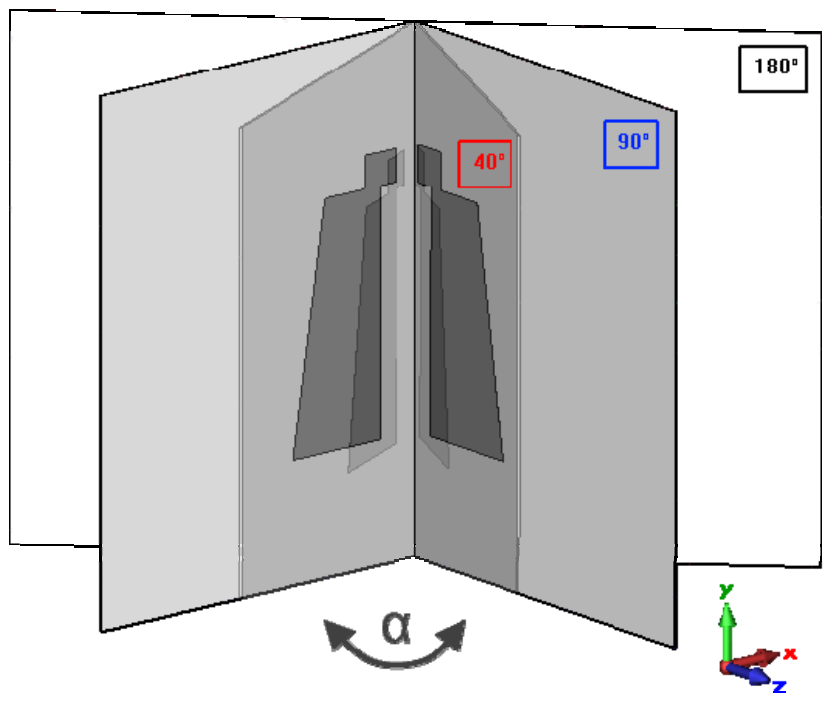

Fig. 5. Angle variation of the dipole arrangement.

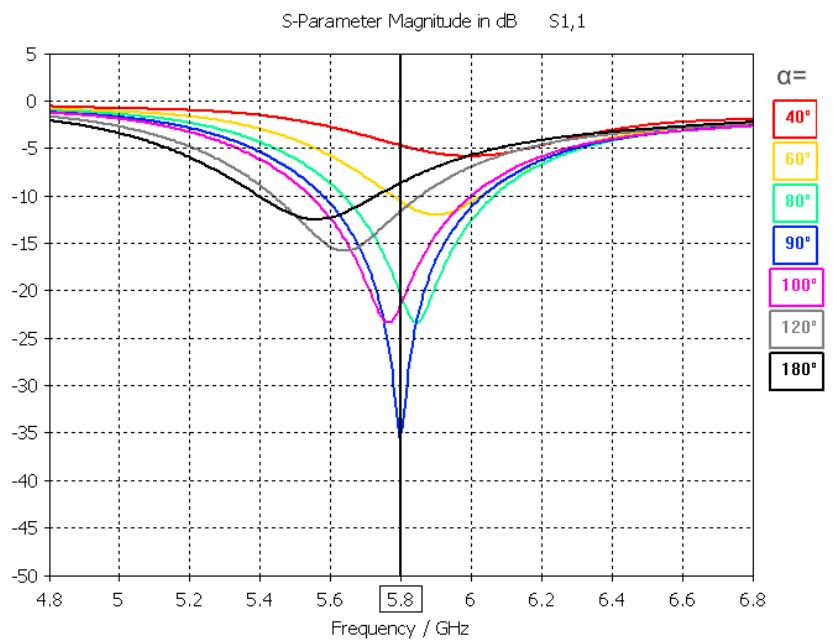

Fig. 6. Scattering parameter S11 in dependency of different orientations (angles).

$6 \mu \mathrm{m}$. Afterwards, the paper substrate was folded in order to receive the three dimensional shape with an angle of $90^{\circ}$ (comp. Figs. 8 and 9).

The shown folding procedure for realizing a three dimensional antenna structure is already described in a prototype study by Mr. Waterhoulse 2007 (Waterhouse, 2007). However, a dipole structure was not realized but a threedimensional folded beverage (spiral) antenna which was not printed and does not have comparable radiation characteristics. His antenna was manufactured lithographically on a flexible FP4 material ( $254 \mu \mathrm{m}$ material thickness).

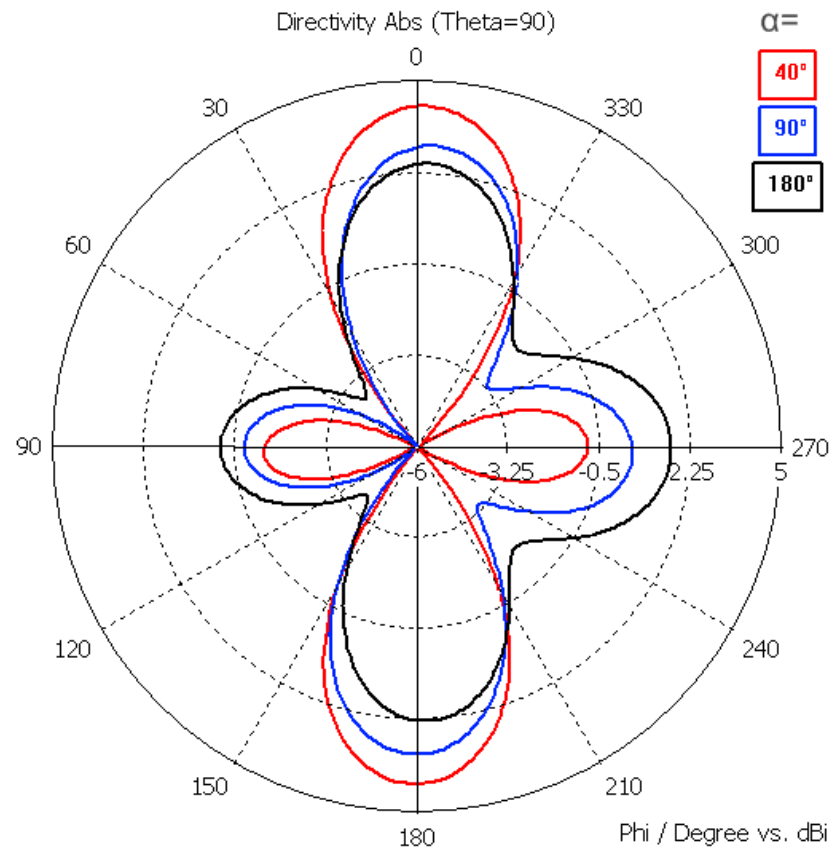

Fig. 7. Directivity polar plots.

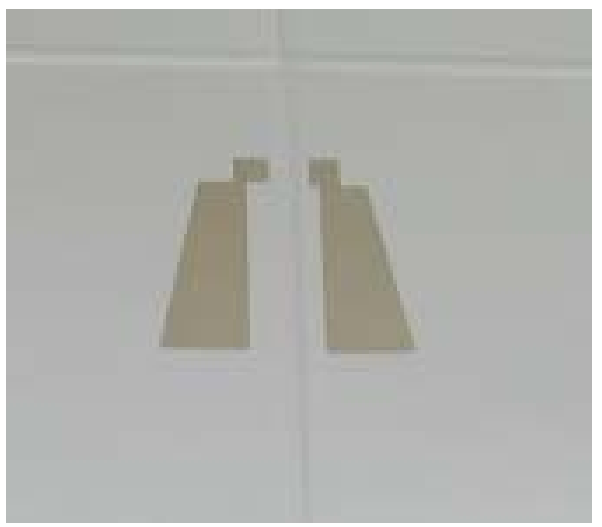

Fig. 8. Planar printed antenna.

\section{Conclusions}

With the development of a 3-D transponder-antenna structure for RFID applications in the frequency range of $5.8 \mathrm{GHz}$, the cornerstone for future RFID applications was successfully laid. Especially with regard to the implemented ability to have a directed communication of the antenna structures, RFID transponder with extended functions such as these of a data transmission node can be utilized. Furthermore, printing the designed antenna structures could be successfully realized. Thereby, it can particularly be highlighted that the antenna structure could directly be printed onto the inside of a cardboard box/transportation container and this box/container was folded in a further processing step and with that the antenna structure obtained its three-dimensional 


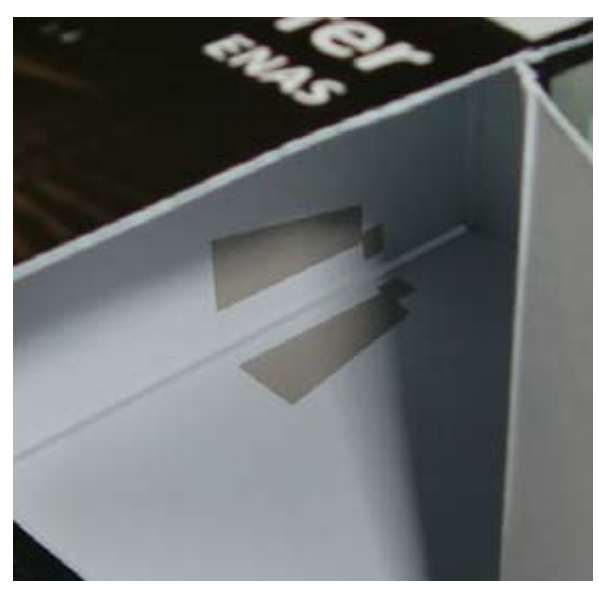

\section{References}

Balanis, C. A.: Antenna Theory, Third Edition, John Wiley \& Sons, pp. 162-184, ISBN: 0-471-66782-X, 2005.

Bullinger, H.-J. and ten Hompel, M.: Internet der Dinge, Springer Verlag, ISBN: 978-3-540-36729-1, 2007.

Waterhouse, R.: Printed Antennas for Wireless Communications, John Wiley \& Sons, pp. 151-159, ISBN: 978-0-470-51069-8, 2007.

Fig. 9. Same as Fig. 8, folded: 3-D antenna.

shape and functionality. Therefore, this antenna structure as well as the manufacturing process is suitable for low cost applications in the logistics and consumer sector as well as a variety of applications for the Internet of Things. 